\title{
CUDA ENHANCED FILTERING IN A PIPELINED VIDEO PROCESSING FRAMEWORK
}

\author{
A Thesis \\ presented to \\ the Faculty of California Polytechnic State University \\ San Luis Obispo
}

\author{
In Partial Fulfillment \\ of the Requirements for the Degree \\ Master of Science in Computer Science
}

by

Austin Dworaczyk Wiltshire

June 2013 
(C) 2013

Austin Dworaczyk Wiltshire ALL RIGHTS RESERVED 


\section{COMMITTEE MEMBERSHIP}

TITLE:

CUDA Enhanced Filtering in a Pipelined Video Processing Framework

AUTHOR: $\quad$ Austin Dworaczyk Wiltshire

DATE SUBMITTED: June 2013

COMMITTEE CHAIR: Professor Christopher Lupo, Ph.D., Computer Science Department

COMMITTEE MEMBER: Professor Alexander Dekhtyar, Ph.D., Computer Science Department

COMMITTEE MEMBER: Professor John Seng, Ph.D., Computer Science Department 


\begin{abstract}
CUDA Enhanced Filtering in a Pipelined Video Processing Framework
\end{abstract}

Austin Dworaczyk Wiltshire

The processing of digital video has long been a significant computational task for modern x86 processors. With every video frame composed of one to three planes, each consisting of a two-dimensional array of pixel data, and a video clip comprising of thousands of such frames, the sheer volume of data is significant. With the introduction of new high definition video formats such as $4 \mathrm{~K}$ or stereoscopic 3D, the volume of uncompressed frame data is growing ever larger.

Modern CPUs offer performance enhancements for processing digital video through SIMD instructions such as SSE2 or AVX. However, even with these instruction sets, CPUs are limited by their inherently sequential design, and can only operate on a handful of bytes in parallel. Even processors with a multitude of cores only execute on an elementary level of parallelism.

GPUs provide an alternative, massively parallel architecture. GPUs differ from CPUs by providing thousands of throughput-oriented cores, instead of a maximum of tens of generalized "good enough at everything" x86 cores. The GPU's throughput-oriented cores are far more adept at handling large arrays of pixel data, as many video filtering operations can be performed independently. This computational independence allows for pixel processing to scale across hundreds or even thousands of device cores.

This thesis explores the utilization of GPUs for video processing, and evaluates the advantages and caveats of porting the modern video filtering framework, 
Vapoursynth, over to running entirely on the GPU. Compute heavy GPU-enabled video processing results in up to a $108 \%$ speedup over an SSE2-optimized, multithreaded CPU implementation. 


\section{Contents}

List of Tables viii

List of Figures $\quad$ ix

1 Introduction 1

2 Background 3

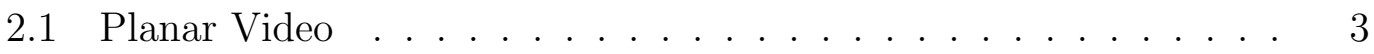

2.2 Byte Representation . . . . . . . . . . . . . . . . 4

2.3 Vapoursynth.................... 5

2.4 CUDA Architecture . . . . . . . . . . . . . . . 7

2.4.1 Thread Block Model .............. 7

3 Design 11

3.1 From Core to CUDA . . . . . . . . . . . . . . . . . 12

3.2 CUDA Performance Optimizations . . . . . . . . . . . . . 13

4 Implementation $\quad 16$

4.1 Lut . . . . . . . . . . . . . . . . . . . 19

4.2 Merge ........................ 20

4.3 Transpose . . . . . . . . . . . . . . . . . . . . 21

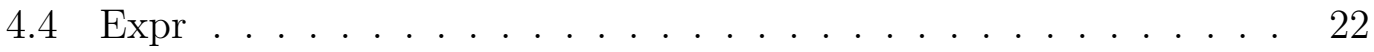

4.4.1 L1 Cache vs Shared Memory . . . . . . . . . . . . 23

4.4 .2 Constant Memory . . . . . . . . . . . . . . 24

4.5 CUDA Streams . . . . . . . . . . . . . . . 25

4.6 Validation . . . . . . . . . . . . . . . . 27 
5 Results $\quad 28$

5.1 Lut Results . . . . . . . . . . . . . . . . . . . . . . . 29

5.2 Merge Results . . . . . . . . . . . . . . . . . . . 30

5.3 Transpose Results . . . . . . . . . . . . . . . . . 31

5.4 Expr Results ....................... 33

5.5 CUDA Stream Results . . . . . . . . . . . . . . 36

5.6 Complex Script Results . . . . . . . . . . . . . . . . . . 40

5.7 Fermi and Kepler . . . . . . . . . . . . . . . . . . . . 41

6 Conclusion $\quad 42$

7 Future Work $\quad 44$

7.1 Multi-GPU Support . . . . . . . . . . . . . . 44

7.2 Extended Filter Support . . . . . . . . . . . . . . . 45

7.3 Expanded Bits Per Pixel support . . . . . . . . . . . . . . . . 45

7.4 Investigate CPU Threading problems . . . . . . . . . . . . . 46

7.5 Providing Support for Non-CUDA Devices . . . . . . . . . . . . . 46

$\begin{array}{ll}\text { Bibliography } & 47\end{array}$ 


\section{List of Tables}

4.1 An overview of the core filters available in Vapoursynth . . . . . 18 


\section{List of Figures}

2.1 An example of banding $[1] \ldots \ldots \ldots \ldots \ldots$

2.2 An example layout of blocks in a grid and each block's associated thread array $[18] \ldots \ldots \ldots \ldots . \ldots \ldots$

2.3 The basic layout of Streaming Machines in the Fermi architecture

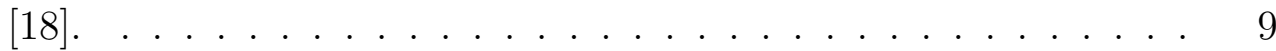

3.1 The effects of containing all shipping logic in one filter versus a filter-by-filter basis. Larger is better. . . . . . . . . . . . . . . 13

4.1 Invert results, with unoptimized and optimized kernels running on the CUDA Kepler and Fermi architectures. . . . . . . . . . . . 17

5.1 Lut execution speed in frames per second. Higher is better. . . . . 30

5.2 Merge execution speed in frames per second. Higher is better. . . 31

5.3 Transpose execution speed in frames per second. Higher is better. 32

5.4 Expr execution speed in frames per second. Higher is better. . . . 34

5.5 Expr execution speed in frames per second for 1 filter iteration. . 34

5.6 Expr execution speed in frames per second for 16 filter iteration. . 35

5.7 Expr execution speed in frames per second for 32 filter iteration. . 35

5.8 CUDA Streams results for one filter iteration on the Fermi architecture. Greater is better. . . . . . . . . . . . . . 37

5.9 CUDA Stream results for one filter iteration on the Kepler architecture. Greater is better. . . . . . . . . . . . . . . . . . 39

5.10 The results of a complex script run on an i7 Xeon E5-2650, i7 3770k, GTX 560 Ti, and Kepler K20Xm GPU. . . . . . . . . . . 40 


\section{Chapter 1}

\section{Introduction}

Video processing (filtering, noise removal, transformation, etc.) is a computationally intensive process, frequently requiring several seconds per frame in the case of extreme motion compensation or similar tasks. Historically, video processing speed has been directly proportional to the sequential processing speed of a single core CPU.

With the move to multiple CPUs, and eventually multicore CPUs, editing and processing speeds improved greatly. This performance boost was eventually counteracted with the move to larger resolution video formats such as 720p, 1080p $(2 \mathrm{~K})$, and eventually $4 \mathrm{~K}$ and $8 \mathrm{~K}$. These massive increases in video resolution led to a heavier and heavier burden on inherently sequential CPUs.

In order to improve multimedia handling in desktop CPUs, chip makers such as Intel and AMD created an instruction set enabling programmers to guide the $\mathrm{CPU}$ in performing a uniform operation, or instruction, on multiple chunks of

pixels, or data, at the same time. This form of parallelism is frequently referred to as SIMD, or Single Instruction Multiple Data. 
These SIMD instructions sets (like MMX, SSE, SSE2, 3DNow!, AVX, etc.)[7][9] often operate on either a 64-bit or 128-bit data block [12]. This instruction width, combined with the 8-bit representation of pixel data in modern video [5], allows for a maximum of 16 pixels to be operated on in parallel by a single core processor. While an excellent improvement over standard sequential processing, it still offers a relatively low performance ceiling, considering that a 1920 x 1080 consists of 2,073,600 pixels.

Recently, an industry shift towards GPU-oriented processing has occurred. GPU architectures offer an alternative approach to video processing. By providing hundreds or thousands of cores, along with a programming and scheduling model aimed specifically at massive compute scalability across all available data, GPUs achieve a high amount of data parallelism. The massively multithreaded approach to general purpose GPU (GPGPU) programming allows for each thread to process one pixel (or more, as covered later in this paper) at a time, completely independent of all other threads. Video pixels are processed by the thousands in GPUs compared to the tens in the case of a standard CPU core.

This thesis details an extension of the Vapoursynth [10] video processing framework that supports a fully GPU-enabled filtering pipeline. It aims to run as much filtering logic on the GPU as possible, resulting in a performance boost of up to $108 \%$ for particular, compute-bound, filter implementations.

All background information will be detailed in Chapter 2, followed by overall design in Chapter 3, implementation in Chapter 4, results in Chapter 5, and conclusion and future work in Chapters 6 and 7. 


\section{Chapter 2}

\section{Background}

Video data is represented in a multitude of formats, which vary in colorspace, resolution, and bit depth. While these formats vary, they are all combinations of several uniform principles, which are described in the following sections. Additionally, the general history and architecture of the Vapoursynth video processing framework are detailed in Section 2.3.

\subsection{Planar Video}

The core of video data is represented by a two dimensional array of pixel values, where each value corresponds to a color or intensity. Video data is commonly represented using three separate planes, and thus three separate arrays. In the case of RGB video, each plane corresponds to red-only, green-only, or blue-only color values. In the case of YV12 video, the base plane corresponds to only luma, or luminance, values, while the two remaining planes correspond to subsampled chroma, or chrominance, values. The chroma planes are subsampled in order to conserve space, and take advantage of the fact that the human eye is more sen- 
sitive to luma information and has trouble discerning variation in chroma-only information [8].

\section{$2.2 \quad$ Byte Representation}

Video formats are not only distinguished by plane count, but by the word size used to represent a pixel value in each plane. Most video formats represent a pixel using only 8 bits [5], or 256 color values. This is done to conserve space, while still providing an acceptable quality image. Professional video is often stored using 9, 10, or 16 bits $(512,1024$, or 65536 color values, respectively) in order to retain more color information and thus a closer representation to the original film content [25]. Most consumer grade equipment cannot play formats with greater than 8 bits per pixel, but this is starting to change as consumers recognize the benefits of an enhanced color range, particularly for computer generated or animated content.

A recent movement in Japan has seen the increased usage of 10-bit video to store anime content [14], as its increased color range prevents a form of visual distortion referred to as "banding" (see the 8-bit gradient in Figure 2.1). Banding is often seen in 8-bit video containing large swaths of solid color or gradients. These large swaths of color are often seen in anime due to its artistic, or drawn, nature, and can be particularly distracting in scenes containing large blank walls or close up shots of character faces.

True 10-bit video playback requires a 10-bit capable display, which is not a common feature in standard desktop monitors. Still, 10-bit video processing is becoming preferred by many encoding experts in the industry, as it provides a greater pixel precision, allowing for greater accuracy in color interpolation and 


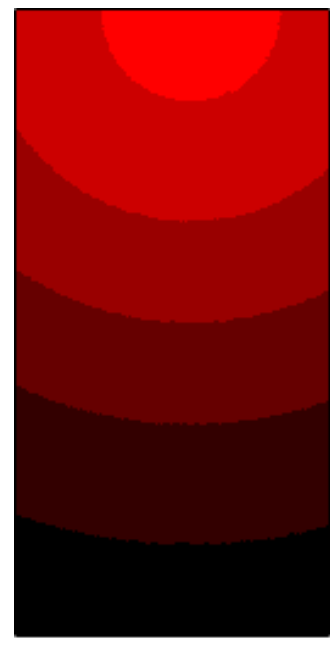

8-bit gradient

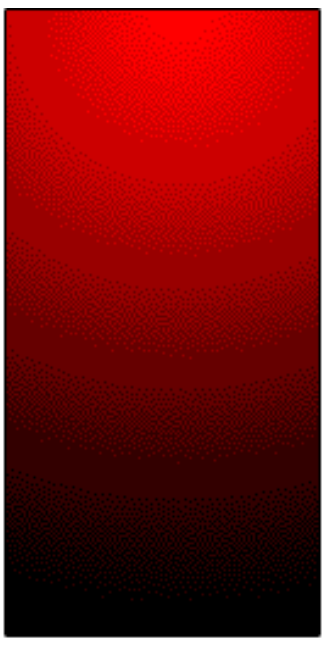

8-bit gradient,

dithered

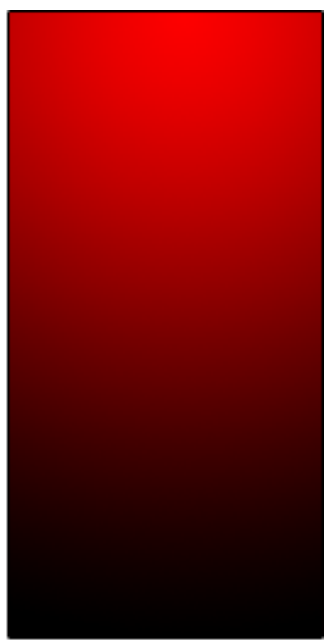

24-bit gradient

Figure 2.1: An example of banding [1].

downsampling.

\subsection{Vapoursynth}

Vapoursynth was written by Fredrik Mellbin [10] and designed from the ground up to be a portable and efficient video filtering framework based off of the much older framework, Avisynth. Avisynth was started back in 2003 by Ben Rudiak-Gould and began as a Windows-only, single-threaded application [23]. Later iterations expanded colorspace support [6] and added multithreading through specialized plugins [24].

Vapoursynth provides a strong API that can be ported to any number of scripting language frontends, including Python, Lua [4], and more. For the sake of simplicity, and the fact that a variety of processing options are already available, audio processing is excluded from initial versions of Vapoursynth. 
Vapoursynth operates through a filter chain paradigm. A video is imported (or created through the use of Vapoursynth's BlankClip() function), and then processed in sequence by any number of filters in a sequential chain. Each frame of the input video is processed by every filter in the order dictated in the loaded script. Additional filters outside of the standard library can be loaded dynamically at runtime, providing a fully modular filtering experience.

This modular approach allows for plugin authors to ignore the specifics of frame loading, frame caching, and even the threading model, and instead focus on strong, optimized algorithms for their plugin implementations.

Additionally, Vapoursynth provides two performance enhancements out of the box: a central threadpool and a frame cache. The thread pool allows for the easy utilization of multicore CPUs. Each thread is assigned to a single frame, which is then pushed through the filter pipeline. After this frame has been processed, it is readied for output in the central core and the processing thread releases its resources and returns to the thread pool to process another frame.

The decision to implement frame-level parallelism helps to keep filter authors from manually managing thread resources. This separation encourages clean and concise code while simultaneously preventing race conditions and nondeterministic bug tracking.

Vapoursynth's frame cache acts as a central list for currently processing frames. By limiting the list size, Vapoursynth can precisely manage its memory usage, preventing large memory consumption spikes. The frame cache is especially useful in the case of temporal filtering plugins, which require specific frames within a radius before or after the current frame. Frames within that radius are likely to be stored in the current frame cache, preventing expensive 
retrieval and processing stalls while a temporal filter waits for dependent frames to be readied.

\subsection{CUDA Architecture}

NVIDIA's Compute Unified Device Architecture (CUDA) [22] embodies a massively parallel computation platform, with a scalable programming model that efficiently adapts to any problem size. The CUDA programming SDK allows for a write once, deploy everywhere approach to data computation, as all computation functions (referred to as "kernels" on the CUDA platform) scale inherently to the capabilities of the NVIDIA device on which they run. It is for this reason that hardware upgrades to a CUDA-capable machine result in a directly proportional performance increase with no code recompilation or additional optimization.

Of course, architectures evolve and certain programming paradigms should be followed in order to take full advantage of all compute devices. A complete understanding of CUDA's thread block model is required to correctly and efficiently optimize GPU kernels, and since the remainder of this paper revolves around the CUDA architecture, a quick summary of CUDA is provided in the following subsections.

\subsubsection{Thread Block Model}

CUDA operates around the idea of a block grid, where each block contains an array of threads (see Figure 2.2). Both grids and blocks can be expressed in 1,2 , or 3 dimensions as long as they do not surpass the limits of the base 


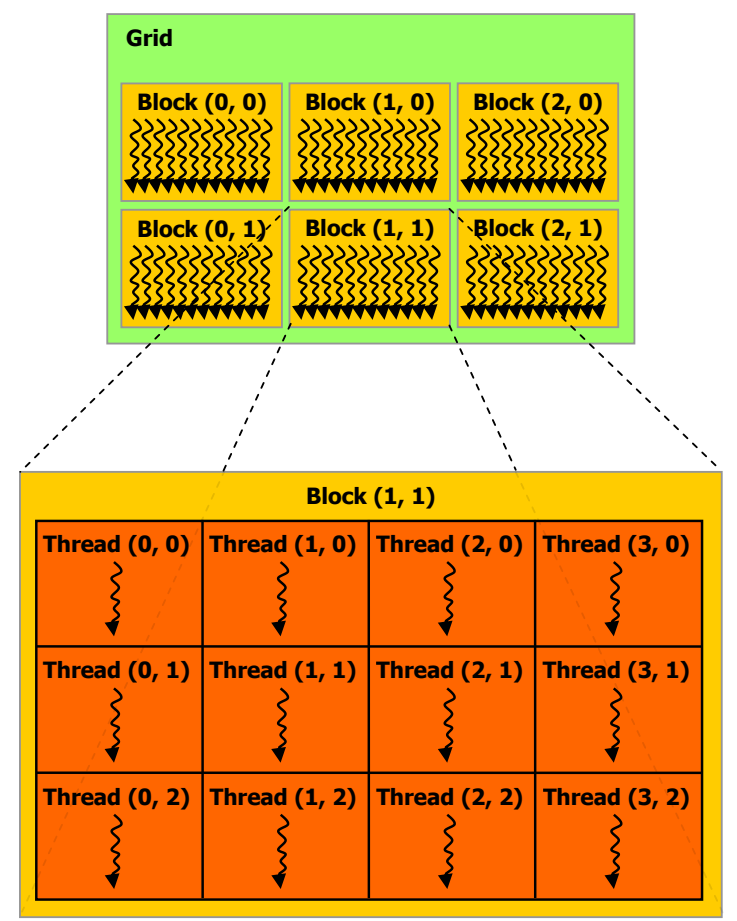

Figure 2.2: An example layout of blocks in a grid and each block's associated thread array [18].

compute architecture. These limits change (generally by growing) from architecture version (commonly referred to as "Computer Capability" (CC) in CUDA documentation) to architecture version, thus requiring a bit of adaptability by the kernel runner when executing. Luckily, CUDA provides support for querying all device capabilities (across multiple devices), enabling kernel executions to be tuned at runtime to a device's CC.

As previously mentioned, each thread block consists of an array of threads. Thread blocks are limited to a maximum of 512 threads for devices less than CC 2.0, and 1024 threads for CC 2.0 and above [15]. Grids have much larger dimensions, with $\mathrm{x}$ and $\mathrm{y}$ dimensions limited to 65535 for $\mathrm{CC}$ less than 3.0, and $2^{31}-1$ for CC 3.0 and above. Block grids are limited to 65535 blocks in the 


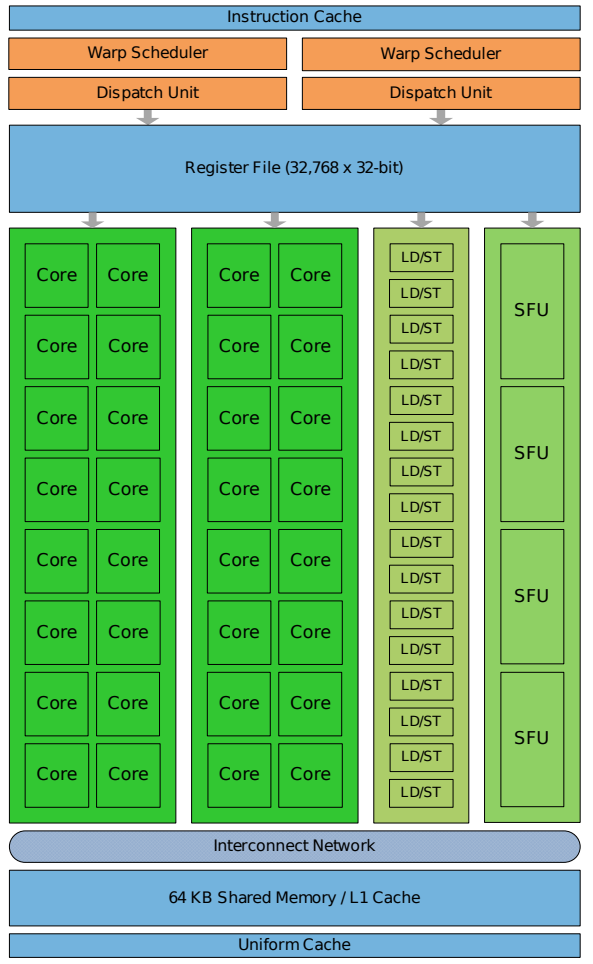

Figure 2.3: The basic layout of Streaming Machines in the Fermi architecture [18].

z-dimension for all current Compute Capabilities.

Threads are executed by Streaming Multiprocessors (SM), which partition and enumerate incoming thread blocks. All threads in a block execute concurrently on a SM, and multiple thread blocks can execute concurrently on a SM. The threads of a block are partitioned into warps of 32 threads and each thread operates in lockstep with the rest of the warp. All threads in a warp execute the same instruction at the same time, thus if a particular thread diverges, the warp executes each alternate code path in a serial fashion. Therefore it is important to reduce branch divergence as much as possible to obtain optimal performance.

CUDA offers a variety of architecture enhancements that provide a strong toolset for programmers to write high performance kernels. These enhancements 
include block-level shared memory, texture and constant memory caches, a variety of atomic and synchronization functions, and much more. All of these enhancements are used in the kernels described in subsequent chapters and will be detailed as needed. 


\section{Chapter 3}

\section{Design}

The goal behind the CUDA port of Vapoursynth is to create a high-performance library of GPU-enabled kernels for use in dynamic video filtering. Additionally, all enhancements are designed to be API compatible and support backwards compatibility with all legacy plugins.

All CUDA kernels are required to be in separate source files, which are externally callable via a $\mathrm{C}$ wrapper. To keep things simple, each filter examines the frame storage location (either host side or device side) of an incoming frame and picks the appropriate processing kernel. If a frame is located in the host's local memory, then a CPU kernel is executed. If the frame is located in the video card memory, then a GPU kernel is executed.

Initial design efforts abstracted out several steps of GPU processing in the core. This was done to encourage additional ports to OpenCL or alternative GPU programming frameworks, preventing Vapoursynth from being locked into CUDA, and thus only NVIDIA GPUs.

During the design phase of this project, a second alternative came to light 
through the GPU Ocelot framework [2], but its utilization is left for future work.

\subsection{From Core to CUDA}

The CUDA enhancement layer is designed to integrate as closely with the core as possible, taking advantage of already written memory management routines, while also enhancing the filtering pipeline to ensure optimal processing speeds when working with GPU data.

A separate memory counter was established in order to allow for the independent tracking of host memory and device memory. Ideally, this will allow for completely separate frame caches, but this is not currently implemented and is intended for future work.

One of the central tenants of GPU processing is to ship all necessary data to the GPU and keep it there as long as possible. This is recommended to reduce the cost of communication over the relatively slow PCI Express bus between the GPU and the host system. If each filter had to ship frame data to and from the GPU every time it's executed, processing would be severely limited to the PCI Express bus speed, instead of the device computation speed. Additionally, the frame transfer logic would be redundant between all GPU-capable filters, as each would require a copy of the transportation code.

In order to prevent the PCI Express bus bottleneck, an additional filter has been added to Vapoursynth's standard library. This new filter has the sole purpose of shipping data to and from the GPU, and must be explicitly called by the user in the loaded script. This central filter contains all frame shipping logic in one place, allowing for optimal PCI Express bus utilization and cleaner filter 


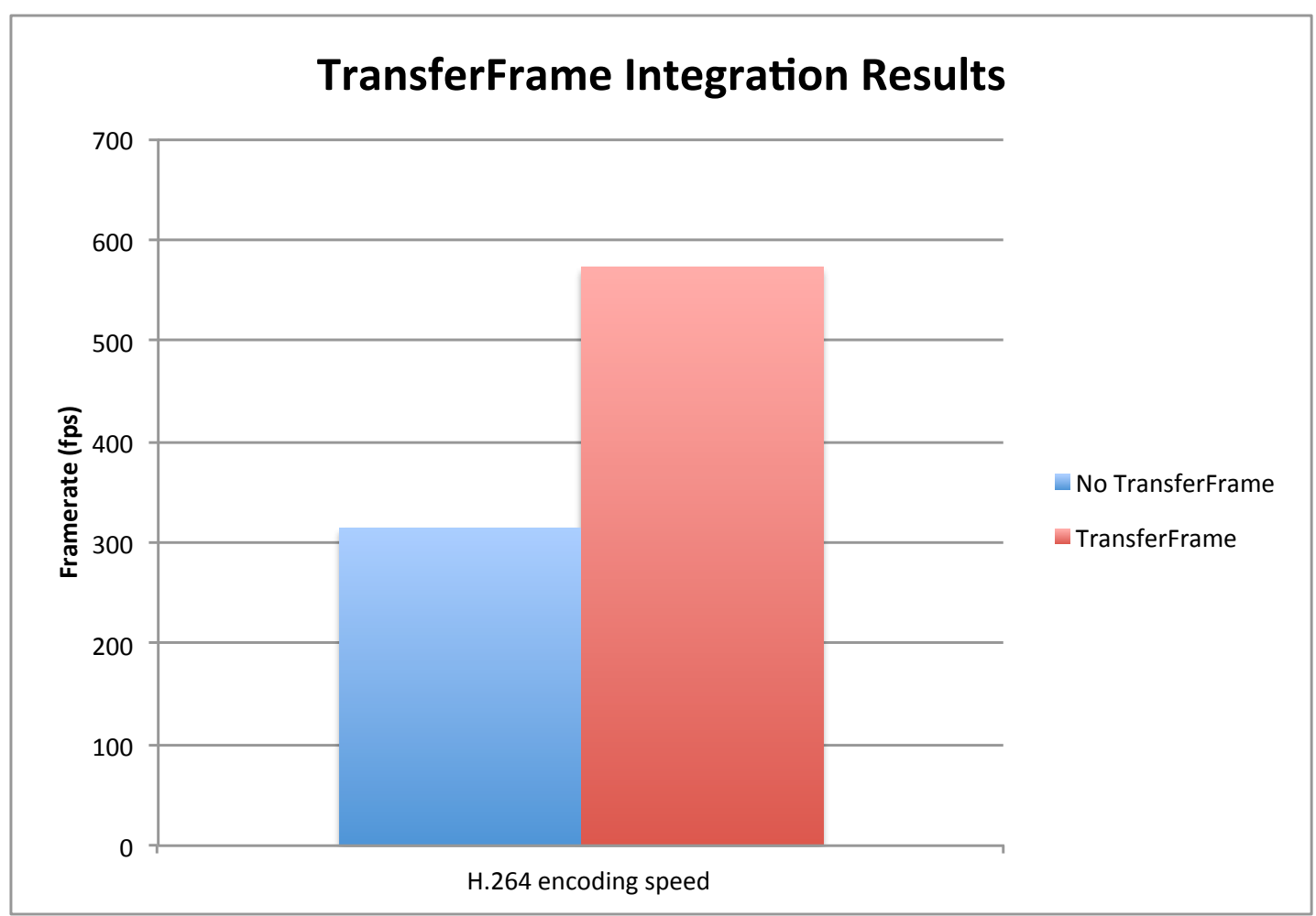

Figure 3.1: The effects of containing all shipping logic in one filter versus a filter-by-filter basis. Larger is better.

code.

The effects of this filter are expressed in Figure 3.1. It is clear that containing frame handling code in one filter leads to a significant performance increase in the filter pipeline.

\subsection{CUDA Performance Optimizations}

Several design considerations are key to ensuring optimal kernel performance for each filter. The first consideration is the aforementioned frame shipping filter, labeled forthwith as TransferFrame. TransferFrame is a user controlled filter who's sole purpose is to ship data to and from the GPU. 
Another design consideration, one that applies directly to every kernel, is coalesced global memory accesses. Global memory coalescing is a key optimization point in CUDA kernels, and is distinctly detailed in the CUDA Best Practices guide [21]. In order to ensure every filter's GPU kernel operates at optimal speed, slight modifications are necessary. These modifications are generally not easy to comprehend at first glance.

For example, a naïve kernel operates on a source plane, with each GPU thread responsible for processing a single pixel. This is a simplistic design that normally works well for matrix data. There is just one problem when it comes to video data that separates it from standard computational mathematics. Standard matrix operations frequently deal with integer or floating point data. Integer and floating point numbers are stored using 4 bytes on CUDA devices. In contrast, most video data is stored in 8 bits, or 1 byte in order to save space. Some professional video is stored in 9,10 , or 16 bits, and very rarely as a float [8]. Due to the standard video pixel taking up just 1 byte, or a fourth of a standard integer, special care must be taken to ensure fully optimized memory accesses on CUDA devices.

On most CUDA devices, with Compute Capability greater than or equal to 2.0, global memory transactions are coalesced along a 128-byte L1 cache line [21]. Assuming a kernel with a block width of minimum 32 threads, and a standard warp size of 32 threads, that means that if the input data is stored in a standard integer format 4 bytes across and each thread requests one integer, the whole warp request coalesces into a single $32 \times 4$ bytes request, aka. 128 bytes.

With consumer video's 8-bit format, this presents a bit of a problem. Since coalescing is concerned with the requests made per warp, and the standard warp size is 32 threads, then a standard memory access of one pixel per thread only requests $32 \times 1$ bytes. This is only a fourth of the standard 128 byte L1 cache 
line, which means that four requests are needed to properly fill an entire cache line. Ergo, a kernel will only operate at around a fourth of its optimal bus speed.

It is possible to work around these access limitations of 8-bit video through the use of pointer casting. By casting the source pointer from an 8-bit integer to a 32-bit (4 byte) integer, memory transactions appear to fulfill the $32 \times 4$ bytes requirement for optimal coalescing. Once the 4-byte integer is retrieved, its contents are recast to four, 1-byte integers, and then processed according to the original filter algorithm. Once all bytes have been processed, the results are recast to a single 4 byte integer and written back to global memory, thus fulfilling the requirements for coalesced reads and writes.

Due to the use of strided memory accesses and uniform block distribution, special consideration must be made when allocating CUDA grids that utilize this casting technique. Strided memory allocation is a programming technique used to optimize lookup performance in large array allocations. Memory is allocated using a unit stride that aligns with a specific device modulus. For example, frames on the $\mathrm{CPU}$ are allocated using a stride of 32 bytes, while they are allocated on the GPU with a stride of 512 bytes. This is done to ensure that all memory allocations align well within the device architecture, instead of being offset and inhibiting read and write performance.

Since each thread is in fact processing four pixels instead of the normal one, the number of required blocks in the $\mathrm{x}$-dimension reduces to a fourth of the original grid size. If a mistake is made and this fact is not accounted for, some pixels will be redundantly reprocessed, effectively wasting GPU resources. Memory allocation strides also need to be compensated accordingly in CUDA kernels, compensating for the fact that the new data source and destination appear to be a fourth as wide compared to their 1-byte counterparts. 


\section{Chapter 4}

\section{Implementation}

The central build system of the Vapoursynth project is Waf, a Python-backed self-coined "meta build system". The first step in CUDA integration was to create a compiler hook for NVIDIA's NVCC compiler in the Waf framework. This hook triggers on all files with the ".cu" file extension, which contain all CUDA code. A few workarounds are required to make the NVCC compiler fully operational with the base Waf configuration script, particularly with respect to compiler symbol definition and debugging symbols. This is due to NVCC's ignorance of standard compiler flags, which must be passed in via a special '-Xcompiler' flag to indicate which compiler flags are to be passed directly to the $\mathrm{C} / \mathrm{C}++$ compiler. Vapoursynth requires several unique $\mathrm{C} / \mathrm{C}++$ flags in order to properly generate its static libraries. These special flags require an extra filtering step during Waf's build configuration phase in order to get NVCC to work properly.

With CUDA compilation working, a test implementation was developed based on the simple Invert filter. The Invert filter accepts an input frame and performs a binary invert on each pixel. Since the actual operation is so concise, with no extraneous code included in the base filter, the Invert filter proved to be a prime 


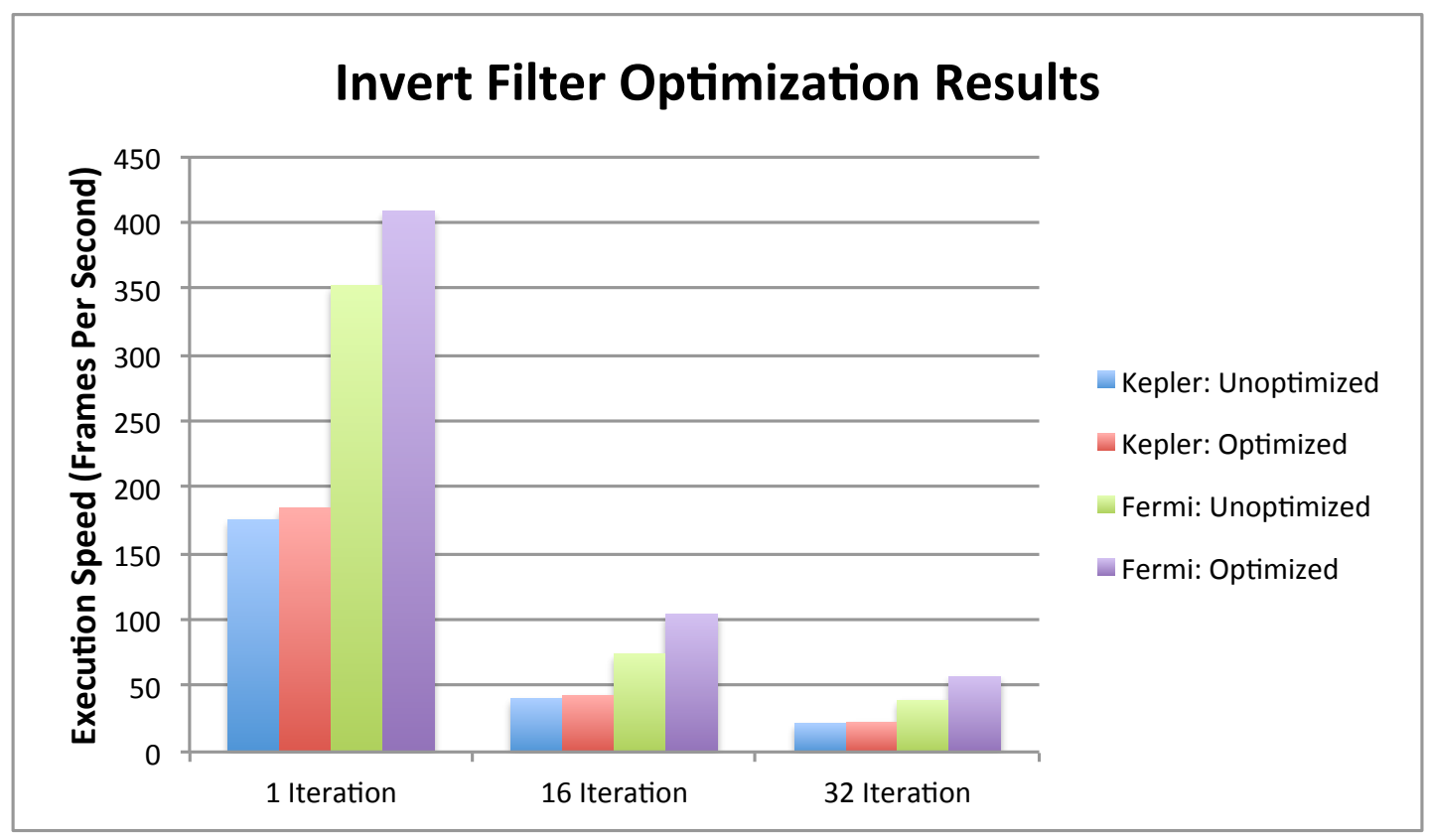

Figure 4.1: Invert results, with unoptimized and optimized kernels running on the CUDA Kepler and Fermi architectures.

proof-of-concept for initial CUDA porting efforts.

With all frame data being managed by the TransferFrame filter, Invert is an extremely simple filter port that requires a basic CUDA kernel and an externally callable $\mathrm{C}$ wrapper. This $\mathrm{C}$ wrapper is used to pass frame data to the GPU kernel from the main processing function. The CUDA kernel is called once for every plane in the input frame, which usually consists of a total of three planes for luma and chroma information, as detailed in Chapter 2.

The kernel follows a form of pixel-level parallelization, where each pixel is operated on independently. Each pixel is read from the source plane, binary inverted, and then stored in the output frame.

An initial version followed a simple design where all threads were assigned to operate on one pixel each, and then write back their results to the destination 


\begin{tabular}{|c|c|c|c|}
\hline AddBorders & AssumeFPS & BlankClip & Cache \\
\hline ClipToProp & CrobAbs/CropRel & DoubleWeave & Expr \\
\hline FlipVertical/FlipHorizontal & Interleave & LoadPlugin & Loop \\
\hline Lut & Lut2 & MaskedMerge & Merge \\
\hline ModifyFrame & PEMVerifier & PlaneAverage & PlaneDifference \\
\hline PropToClip & Resize & Reverse & SelectClip \\
\hline SelectEvery & SeparateFields & ShufflePlanes & Splice \\
\hline StackVertical/StackHorizontal & Transpose & Trim & Turn180 \\
\hline
\end{tabular}

Table 4.1: An overview of the core filters available in Vapoursynth.

frame. As discussed in Section 3.2, this is a very inefficient design for the CUDA architecture and provides relatively low throughput. A second, more efficient kernel using casting for coalesced memory accesses was created. A comparison of the execution speed between the two implementations is provided in Figure 4.1. Results were tabulated over three separate runs, where each run executed the Invert filter 1, 16, or 32 times. Additionally, all runs were executed on both a Fermi and Kepler NVIDIA GPU. The details behind these cards are provided in Chapter 5 .

After successfully implementing a working Invert filter, work began on porting the standard filter library of Vapoursynth. At the time of this writing, the standard library consists of the filters detailed in Table 4.

Among the standard set of filters, there are a few that actually have no effect on frame data, and thus require no extra work to provide a GPU-compatible version. Several other filters (such as AddBorders, BlankClip, and SeparateFields) only perform basic memory set or copy operations, and thus provide little ground for GPGPU research.

It is for this reason that a subset of the standard filter library was selected for implementation. This subset provides a variety of video processing method- 
ologies, which help to demonstrate the strengths and weaknesses of GPU-based video processing, as well as emphasize differences between CUDA architecture generations.

The following sections break down each ported filter and explain its strengths and weaknesses, along with a comparison between its CPU and GPU algorithms.

\subsection{Lut}

The Lut, or look-up table, filter is one of the simplest filters in the ported set. The look-up table is precomputed, containing $2^{B}$ entries, where $B$ is the number of bits per sample in the source frame. The filter operates by performing a simple look-up using the value of a source pixel as an index. The value contained in the look-up table for the given source pixel is then written back to the destination frame.

This boils down to two global memory reads with one global memory write per pixel. Since there is essentially no arithmetic computation in the Lut filter, it becomes entirely memory bound. Initial thoughts for optimizing the Lut filter include loading the look-up table into a different GPU memory, such as constant or shared memory. Unfortunately, due to the look-up table's unknown size until runtime, these efforts are problematic.

Loading the look-up table into shared memory is essentially impossible given

the fact that there are only $48 \mathrm{~KB}$ of shared memory per block in CUDA. If an input clip contains 16 bits per pixel, the look-up table requires $2^{16}$ entries, equaling 131,072 bytes or $128 \mathrm{~KB}$, which is well over shared memory's size limit.

Constant memory may be a possible alternative, but speed increases may 
be limited due to constant memory's broadcast architecture. If all threads in a warp access the same memory location, then the memory accesses coalesce and the resulting look-up is broadcast to the entire warp. However, any requests with differing addresses are serialized, therefore minimizing the advantages of constant memory.

Additionally, constant memory must be allocated at compile time, not execution time. Since a look-up table can be a variety of sizes, most of which are well over constant memory's $64 \mathrm{~KB}$ size limit, constant memory is not a valid containment location.

\subsection{Merge}

The Merge filter takes in two clips and produces a blended combination of the two. A configurable floating point bias can be passed in to indicate a preference towards one clip or the other, with the default value being 0.5 , resulting in equal parts being merged from the two input clips.

With two input clips and one output clip, the Merge filter, like Lut, requires two global memory reads with one global memory write. It stands apart from Lut in the fact that it requires an arithmetic operation for an output pixel to be produced. Granted, it is a relatively simple arithmetic operation, so Merge is still primarily memory bound, but it begins to tip the scales towards a computationally bound filter. 


\subsection{Transpose}

Vapoursynth's Transpose filter performs a standard matrix transpose operation on an input frame. There is no arithmetic operation necessary to render a pixel value, simply a global memory read and a global memory write. However, Transpose differs from other filters such as Merge and Lut in the fact that it requires the use of shared memory for optimal GPU performance. Transpose also requires particular algorithmic adjustments to compensate for the fact that each GPU thread operates on four pixels at a time while still providing coalesced memory accesses for both reads and writes to global memory.

In order to achieve optimal memory performance, particularly on memory writes, a large block of shared memory is allocated to store intermediate pixel values. Each 4 byte chunk of pixels is read in from the source frame by a GPU thread. With all threads working together, memory accesses coalesce into a single unit as described in section 3.2. This works well for read in, but requires an adaptation for write out. The reason for this is that a single thread attempting to write a row of four pixels to a column of four pixels (as required by definition of a transpose) causes significant memory thrashing for each additional pixel. This stems from the fact that each pixel in a column is separated by the frame's unit stride in memory. Thus, writing four pixels in a column requires four separate memory transactions.

This performance problem can be surmounted using shared memory and a different thread organization model for write back. In short, the new algorithm has a thread read in a row of pixels and write back a row of pixels. This contrasts to the standard approach, in which a single thread reads in a row of pixels and writes back a column of pixels. 
In order to achieve coalesced memory accesses for both read and write, use of CUDA's synchronization function,___syncthreads(), is required. _-_syncthreads() is CUDA's native, block-level synchronization call which ensures that all threads in a block reach the same code point at the exact same time. The use of _-syncthreads() and shared memory ensures that all threads have loaded the necessary data into shared memory before proceeding to the write back phase. This prevents threads from possibly writing back uninitialized values to global memory.

Once all threads have reached the synchronization point, they proceed to write back values to the same relative global memory addresses they read from. The difference is the manner in which shared memory is accessed. On read, shared memory is accessed in the same $\mathrm{x}$ and $\mathrm{y}$ coordinates as the thread's relative position in the thread block. On write back, the $\mathrm{x}$ and $\mathrm{y}$ coordinates are flipped, and due to shared memory's high performance architecture, no performance hit occurs during the column-wise memory access.

\subsection{Expr}

Where Lut, Merge, and Transpose tend to be memory-bound filters, Expr has the potential to be almost entirely compute-bound due to its purpose as a reverse Polish notation evaluator. Expr operates by accepting a reverse Polish notation string, which contains references to source pixels as well as standard mathematical operations such as addition, subtraction, square root, log, and many more [11]. Additionally, Expr supports basic ternary operators and "greater than" or "less than" operators for more complex processing.

The CPU version of Expr was written by Fredrik Melbin [11], and includes 
a fully optimized SSE2 assembly implementation. Expr works with an internal stack for operand processing, in addition to a second stack for operation tracking and execution. The operation stack is evaluated instruction by instruction and corresponding results are stored on the internal operand stack.

Expr is a rework of Avisynth's MaskTools, specifically mt_lut, mt_lutxy, and mt_lutxyz. It is for this reason that Expr supports expressions utilizing up to three separate input clips, which allows for highly specialized filtering techniques such as edge isolation, sharpening, and much more.

The GPU version of Expr utilizes several import components of the CUDA architecture and demonstrates a significant performance boost over its CPU version, even when factoring in the frame transfer overhead to and from the GPU.

Expr's optimization techniques are described in detail in the following sections.

\subsubsection{L1 Cache vs Shared Memory}

Expr, like Lut, Merge, and Transpose, operates on a block of four pixels per GPU thread, which helps to meet coalescing requirements. However, Expr requires a special operand stack per thread, where operation results are stored for future processing. This operand stack is represented as an array of floating point numbers. Unfortunately, due to thread-level register allocation in CUDA (which is governed by the NVCC compiler at compile time), this stack cannot be safely stored in ultra-fast registers, and instead spills over to what CUDA calls local memory.

Local memory is actually the exact same thing as global memory in CUDA, only accesses are restricted per thread. Local memory is used whenever local 
variables in a CUDA kernel cannot be stored in registers. Luckily, CUDA caches local memory accesses via the L1 cache, which helps to significantly reduce lookup latency and encourages a high performance kernel. CUDA's L1 cache shares its precious resources with shared memory, with the default configuration providing $48 \mathrm{~KB}$ of space to shared memory and a mere $16 \mathrm{~KB}$ to the L1 cache.

The current implementation of Expr does not require any shared memory, but it does require heavy use of the L1 cache due to its operand stack spillage. In order to combat the performance hit of this stack spillage, CUDA offers the ability to configure the cache configuration on runtime via the cudaFuncSetCacheConfig() function. Using this function, it is possible to reverse the cache bias towards shared memory and instead provide the L1 cache with 48KB and shared memory with a mere 16KB. This increased cache space results in a measurable speed up in kernel execution, as most, if not all, local memory accesses can be cached in L1, preventing the significantly slower accesses to global memory.

\subsubsection{Constant Memory}

Expr operates by pulling from a set of operation instructions, which do not change during the life of the kernel and are required by all threads in a warp to operate. It is these properties that make Expr's operation instructions a prime target for use in constant memory.

Constant memory provides two distinct advantages to Expr's kernel execution which result in a marked performance speedup. The first is the architecture of constant memory, which is essentially a specialized, read-only data cache that operates at a much lower latency than global memory.

The second advantage that constant memory provides is its unique broadcast 
capabilities. If the same memory address is requested from constant memory by all threads in a warp, only one look-up occurs with the resulting value broadcast to all threads of the warp. This saves on memory bandwidth and kernel wait time, allowing Expr to continue performing arithmetic operations as quickly as possible.

These optimizations reduce Expr's dependency on memory operations, enabling it to instead focus on pure arithmetic throughput, for which CUDA was specifically designed. The effects of these optimizations, along with the effect that different CUDA architectures have on arithmetic throughput, are detailed in Chapter 5.

\subsection{CUDA Streams}

To further encourage processing parallelism, CUDA provides a framework for asynchronous kernel execution with respect to the host. This allows the CPU to schedule kernel executions and then continue on with its host-side work. Almost all functions in the standard CUDA library have an asynchronous implementation, and often only require one additional parameter. This additional parameter is the CUDA Stream identifier.

Streams are CUDA's way of organizing simultaneous memory transfers or kernel executions. The number of streams that can be executed at the same time depends on the Compute Capability of the GPU. At the time of this writing, CC 2.0 and higher support the use of streams, with CC 2.0+ supporting up to 16 concurrent streams and the new CC 3.5 supporting up to 32 concurrent streams [20]. 
CUDA stream support was implemented in the GPU port of Vapoursynth within the first few versions of the core framework. Early versions assigned streams per frame via the TransferFrame filter, using a frame-specific property. TransferFrame simply stored a stream index value in the frame property which could then be used to retrieve a stream reference from a central pool. This central pool is allocated once at script startup, with all streams recycled every 16 or 32 frames, according to device Compute Capability. Every GPU-enabled filter retrieved the stream index from the incoming frame's properties. The stream index would then be used to retrieve a stream reference from the central pool, allowing for fully asynchronous kernel launches within the current filter context.

Later versions kept the stream pool but instead assigned streams on a per plane basis. This removed the need for a frame property, and moved a lot of the stream handling into the core, away from the filter developer. Additionally, while earlier versions used synchronous memory copies between the host and device, this new version allows for completely asynchronous memory copies per plane. The Kepler architecture is especially adept at utilizing streamed kernels and memory transfers due to its new HyperQ scheduler. HyperQ was introduced with the new GK110 Kepler architecture [19] and allows for 32 simultaneous hardware-managed connections (compared to the single connection available in Fermi). Each stream is handled by its own hardware work queue and inter-stream dependencies are optimized, with operations in one stream no longer blocking other streams. The effects of HyperQ are detailed in Chapter 5. 


\subsection{Validation}

While high performance kernels are an important aspect of this thesis, high performance is only useful if it's programmtically correct. In order to ensure correctness, a suite of Python unit tests were created. Each unit test evaluates a filter, with several filters having multiple unit tests for a variety of parameter configurations. A unit test operates by running a generated input clip under both the CPU and GPU implementations of a target filter. Next, every plane of every frame is compared in the resulting output clips. If the absolute difference between two versions of a plane is greater than zero, the test fails.

All currently implemented filters posses a corresponding unit test, and all tests pass with 100\% bit identical output between the CPU and GPU filter algorithms. This is extremely usefull for framework development and filter refinement, as all output changes are immediately testable. 


\section{Chapter 5}

\section{Results}

Results were collected on two separate CUDA architectures, Fermi and Kepler. The Fermi card is a GTX 560 Ti by MSI [3], with 1024 MB of GDDR5 RAM and a 256-bit wide memory bus. The Kepler card is a K20Xm [16], with 6144 MB of RAM and a 384-bit wide memory bus, with error-correcting code (ECC) memory enabled. All CPU tests were conducted on an Intel Core i7 3770k, running at $3.5 \mathrm{Ghz}$ with a $3.9 \mathrm{Ghz}$ Turbo boost, with $16 \mathrm{~GB}$ of DDR3 RAM.

All tests were run using a custom Python framework, with all filters run in a serial fashion using increasing thread counts, iteration counts, and CPU or GPU processing. Results are then written back to a CSV file and tabulated in the following graphs.

Each filter is run with 1, 2, 4, and $8 \mathrm{CPU}$ threads. This provides a direct comparison between GPU code and several forms of parallelized CPU code. Additionally, all filters were run with 1, 16, and 32 iterations. A larger iteration count reduces the overhead of CPU to GPU transfers over the PCI Express bus, demonstrating the performance benefits of GPU code under a computationally heavy 
workload. All iterations utilized a 1920 x 1080 resolution clip consisting of 1000 frames. The clip was generated in the CPU host memory using Vapoursynth's BlankClip() function.

GPU results are reported using only one CPU thread, as performance degrades with each additional CPU thread when operating on a purely GPUenabled filter pipeline. Additional research needs to be conducted as to why this performance degradation actually occurs. Nevertheless, the greatest performance for GPU code occurs when the least amount of CPU resources are used, resulting in very favorable results from a system overhead standpoint. With the filter chain using the least amount of CPU cycles possible, the rest of the processor can focus on alternative tasks, such as H.264 compression or high resolution video playback.

\subsection{Lut Results}

As detailed in Chapter 4, Lut is a memory-bound kernel meaning that its performance is on par with that of a memory copy operation, with a few caveats. Lut's GPU performance, as detailed by Figure 5.1, shows a lower execution speed when compared against all other CPU runs for 1 iteration. This is due to the simple fact that all data must be shipped to the GPU before it can be operated on, as well as shipped back for output. This performance limitation is reduced when multiple iterations occur, as seen in the 16 and 32 iteration runs.

Lut's 16 and 32-iteration performance numbers indicate a more favorable performance profile, as they consistently beat out the 1 and 2 CPU thread implementations. The GPU version falls a bit below the numbers achieved by the 4 and $8 \mathrm{CPU}$ thread runs, although only by a small margin. It is important to 


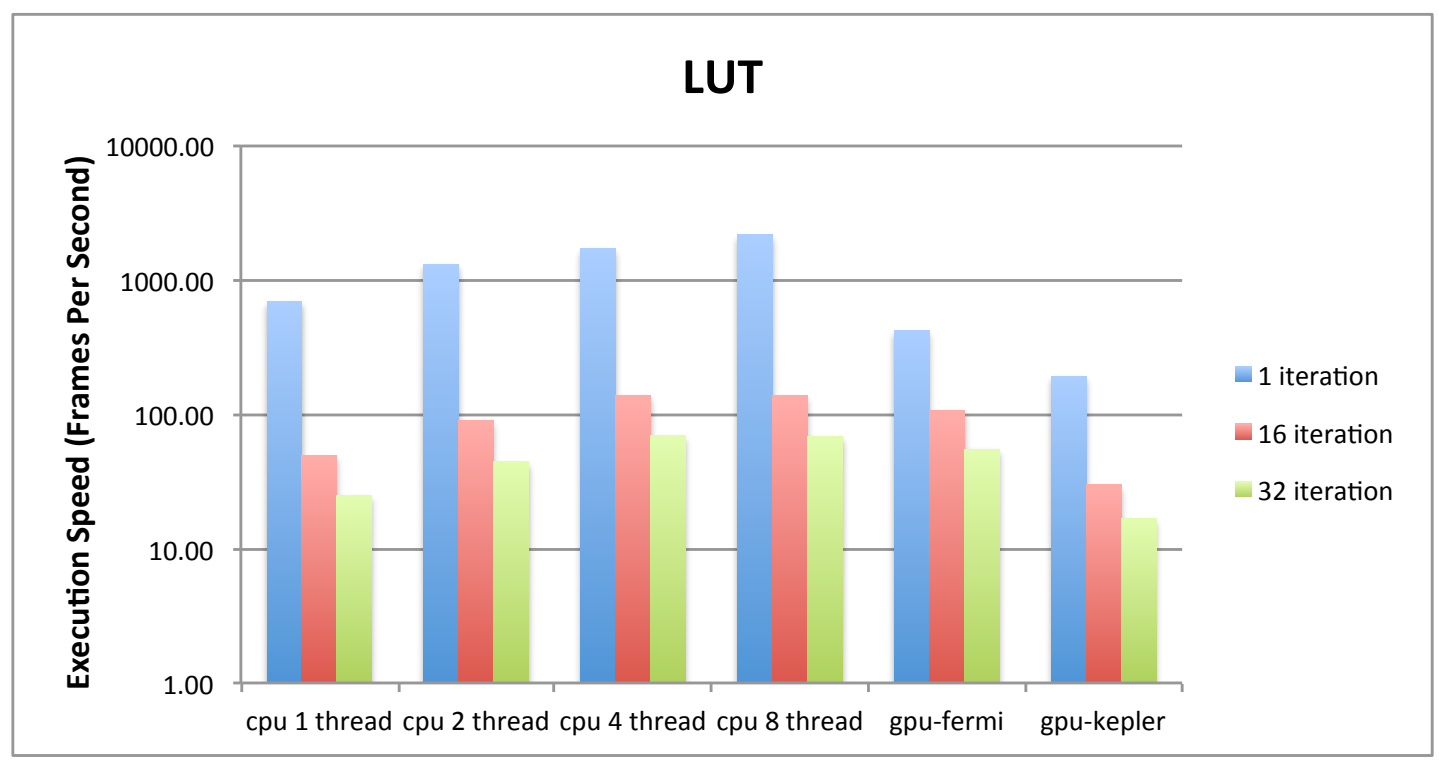

Figure 5.1: Lut execution speed in frames per second. Higher is better.

remember that the GPU version of LUT is using only a 4th or an 8th of the CPU resources of those latter $\mathrm{CPU}$ runs, while still achieving a comparable result.

\section{$5.2 \quad$ Merge Results}

Merge shares similar results with Lut, as it too is a memory bound filter. As detailed in Chapter 4, Merge does requires some basic arithmetic in order to produce its output frame. These extra arithmetic instructions result in a slightly reduced throughput when compared to Lut, as more time is spent performing actual computation instead of a pure memory manipulation operation.

Merge's single threaded performance for one iteration is almost twice as slow for the GPU algorithm when compared to the CPU algorithm (see Figure 5.2). This performance gap only increases for every additional thread applied to the CPU implementation, which is to be expected. However, when multiple iterations 


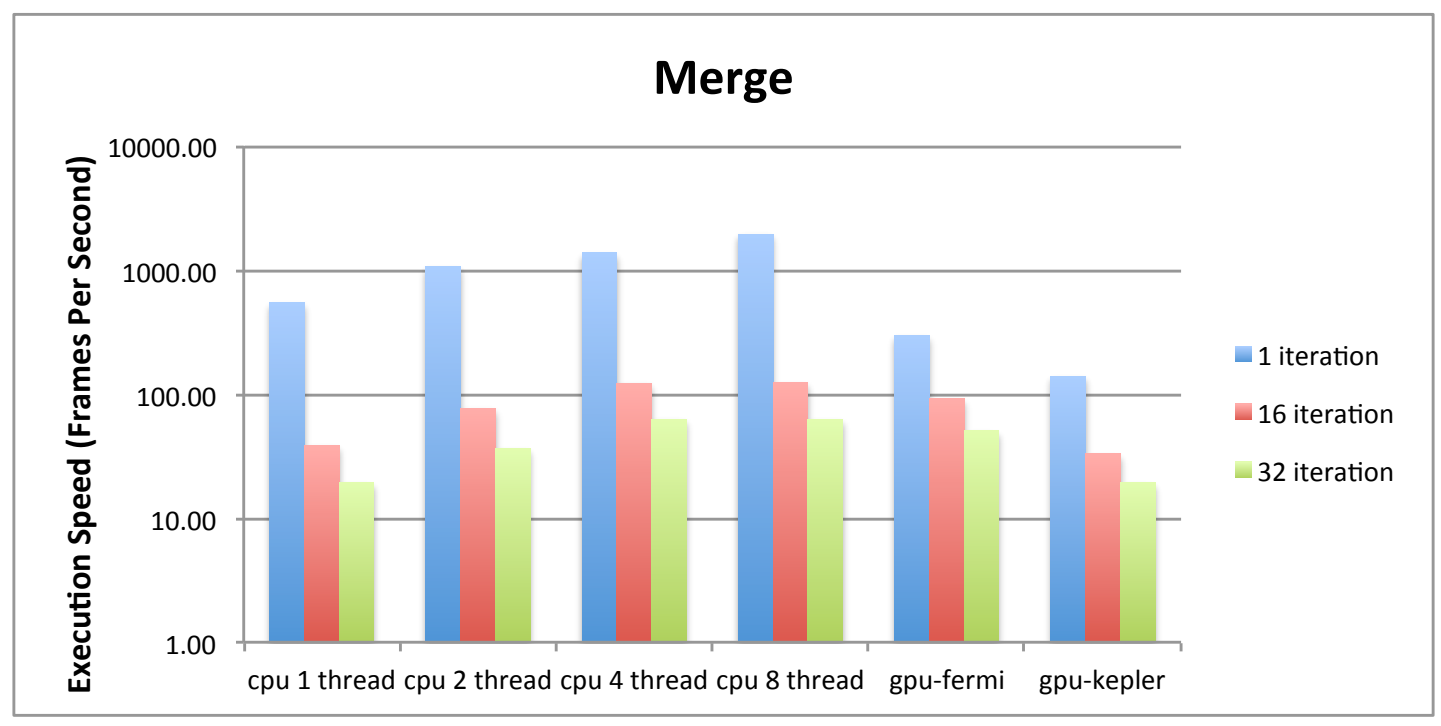

Figure 5.2: Merge execution speed in frames per second. Higher is better.

are introduced, the GPU version of Merge gains some ground back by beating out the one and two thread CPU implementations. It can't quite beat the four and eight thread CPU implementations, but the delta is significantly smaller than the single iteration execution. Again, it is important to keep in mind that the GPU version of Merge only uses one CPU thread, so for all iterations the GPU version of Merge achieves greater or comparable performance using only a fraction of the CPU.

\subsection{Transpose Results}

Transpose is an interesting filter and compares well against both Lut and Merge as a memory bound filter. As shown in 5.3, Transpose demonstrates a level of performance between that of Lut and Merge. This is likely due to Transpose having a much greater instruction count than Lut, while also not requiring any additional arithmetic to render an output pixel, as in the case of Merge. Yet 


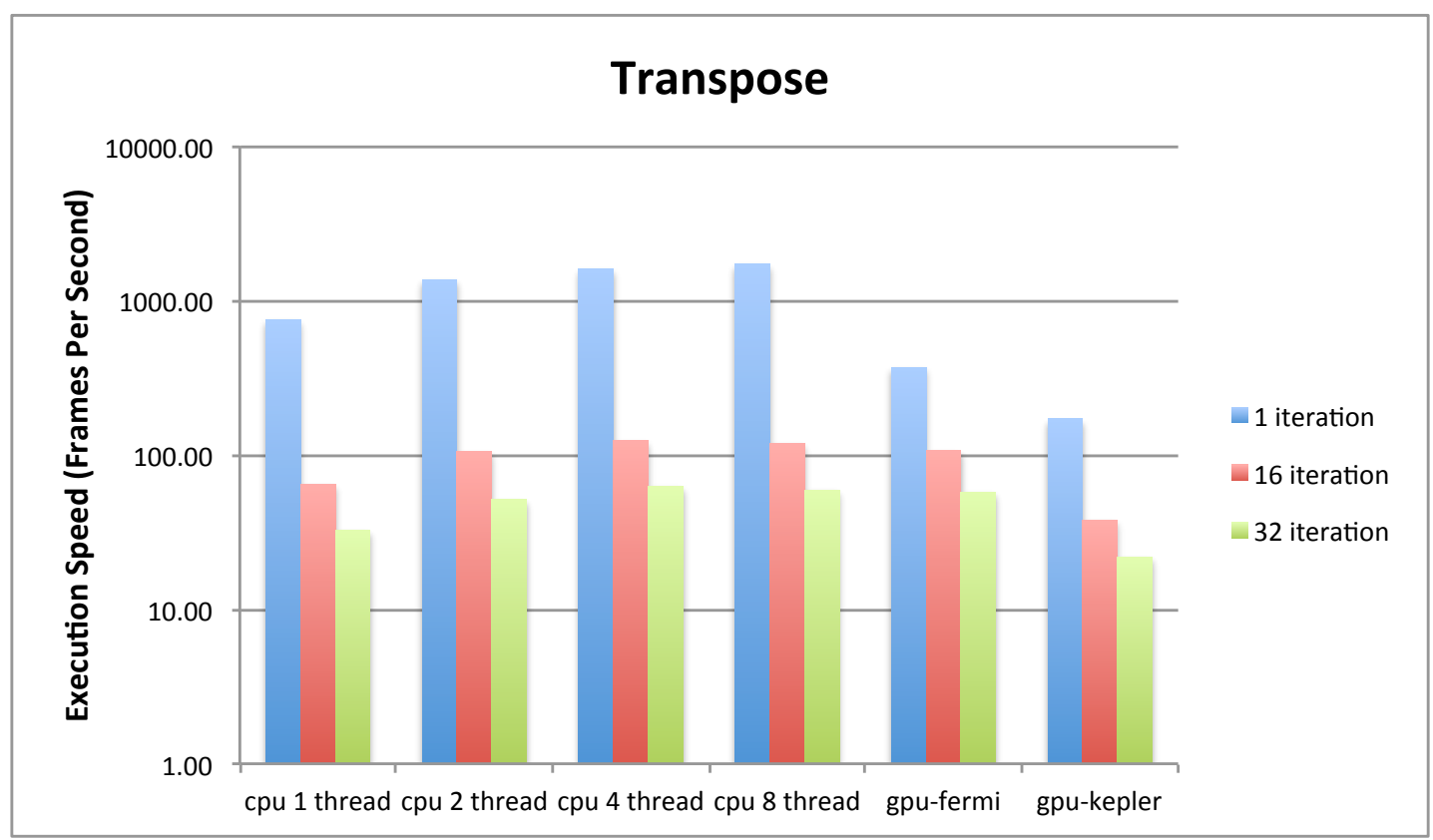

Figure 5.3: Transpose execution speed in frames per second. Higher is better.

again, as with all memory bound filters, the GPU version of Transpose trails behind the CPU version for all single iteration tests. Since the actual kernel execution time on the GPU is so small, Transpose's performance is governed almost entirely by the transfer speed between the CPU and the GPU along the PCI Express bus.

Interestingly, Transpose demonstrates a performance even greater than Lut when operating with 16 or 32 iterations. Even with Transpose's increased instruction count, it only requires one global memory read and one global memory write per pixel, whereas Lut requires two global memory reads for every global memory write. With heavier iteration counts Tranpose becomes a high throughput filter due to its simple memory reordering. Lut and Merge require extra memory reads or arithmetic operations in order to render an output pixel and thus suffer some delay between pixel read and pixel write. 


\subsection{Expr Results}

Expr is juxtaposed from the three previous filters in the fact that it is a compute-bound filter, meaning that most of its time is spent performing arithmetic calculations instead of memory lookups. It is for this reason that the GPU version of Expr demonstrates a significant performance increase over the original CPU version, even when using an SSE2 optimized algorithm.

This performance increase stems from CUDA's throughput oriented cores, which excel at performing raw numerical computation. CUDA cores are generally much simpler than standard x86 processors, as x86 processors are optimized for sequential code performance and use a variety of more complex architectural features such as increased cache sizes, complex branch prediction, and an emphasis on instruction pipelining. CUDA cores are much simpler with smaller cache sizes, simple memory models, and a design emphasis on massively parallel applications such as graphics or geometry calculations. It is for this reason that CUDA cores excel at raw number crunching when juxtaposed with an x86 processor.

Figure 5.4 details Expr's CUDA performance against a multithreaded, CPU SSE2-optimized algorithm. It is quite clear that the CUDA version of Expr offers a significant performance increase over the CPU version, with the Kepler's 32 iteration (Figure 5.7) run even beating out several 16 iteration (Figure 5.6) runs on the CPU in pure frames per second. In fact, when comparing single iterations (Figure 5.5), the CUDA version of Expr demonstrates a 108\% performance improvement over the SSE2-optimized CPU implementation when running on the Fermi architecture, and a $68 \%$ performance improvement when running on the Kepler architecture.

Expr's performance is largely governed by it being a compute-bound filter. 


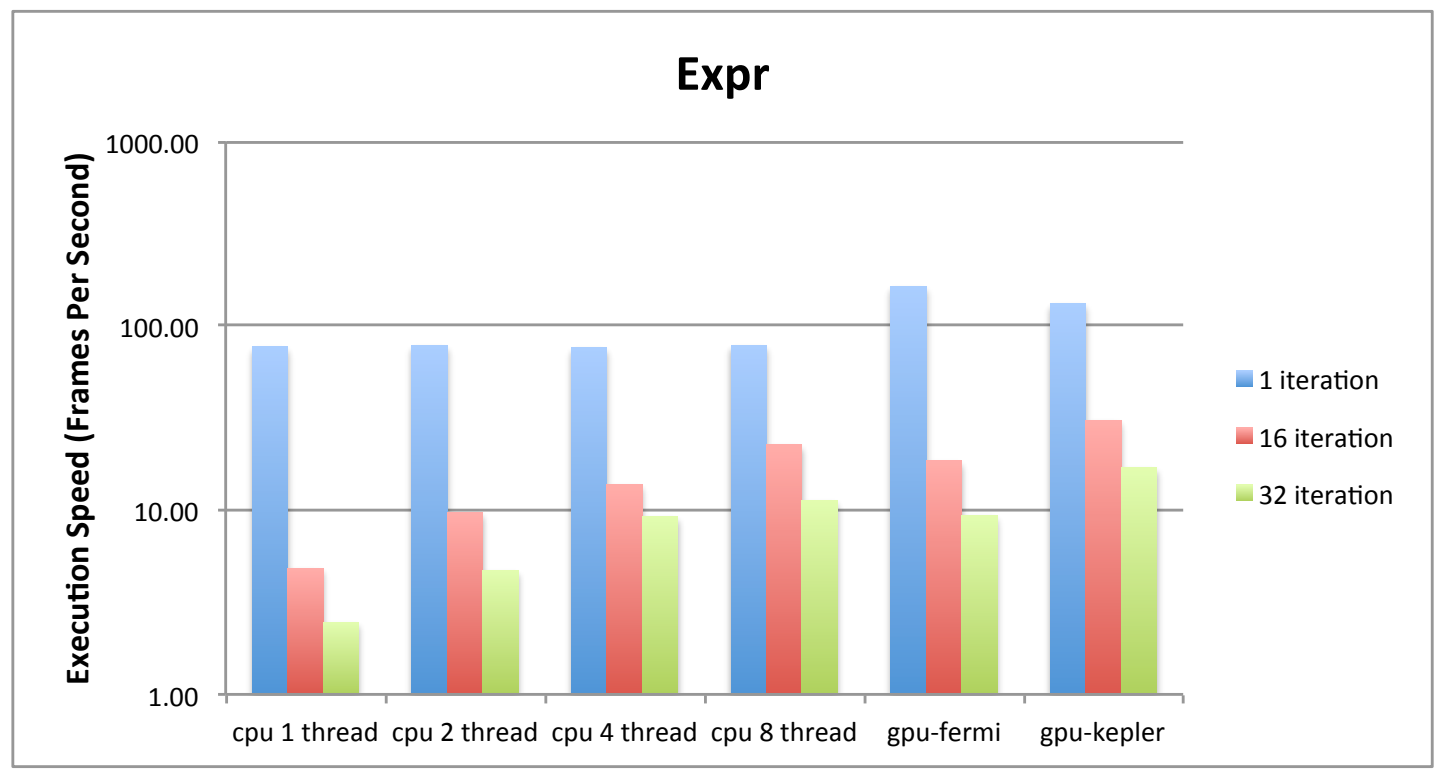

Figure 5.4: Expr execution speed in frames per second. Higher is better.

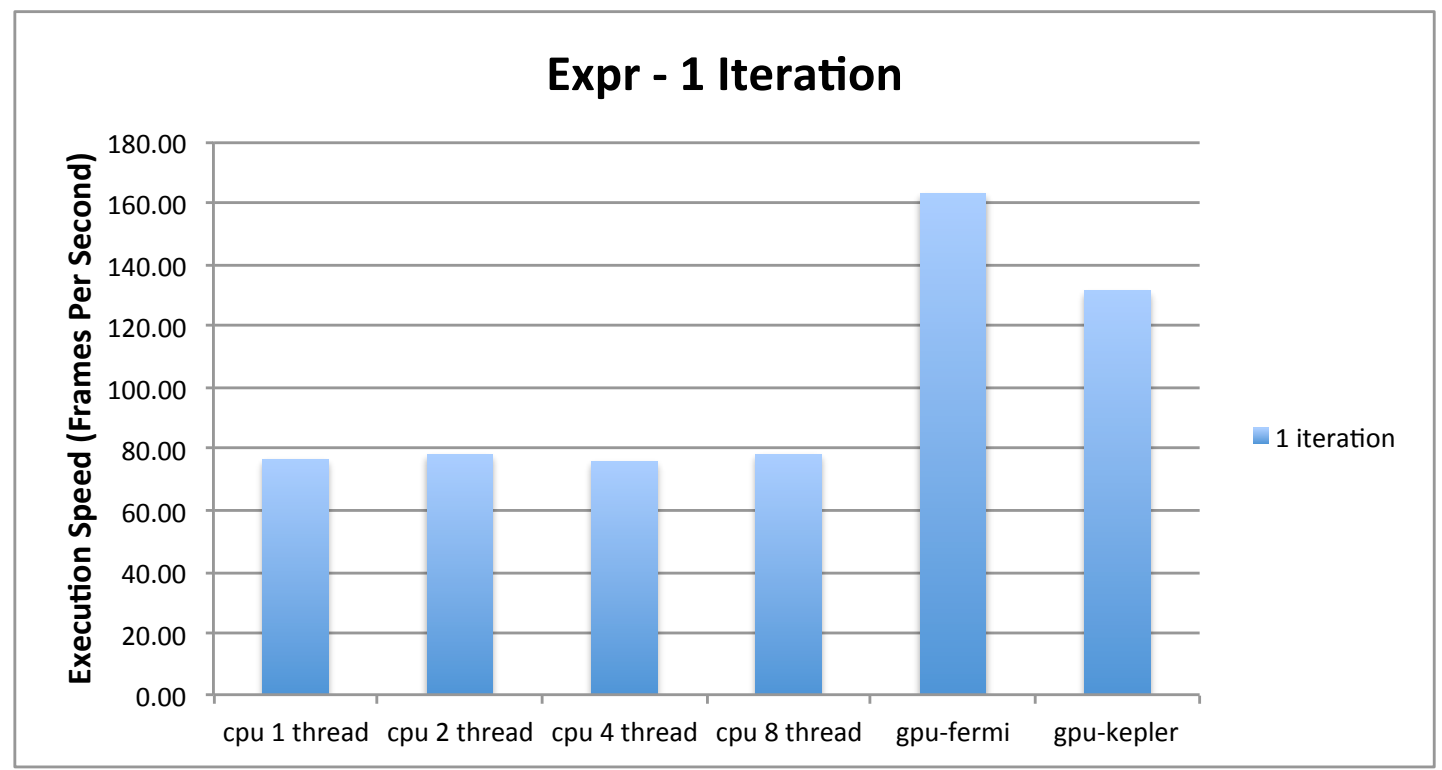

Figure 5.5: Expr execution speed in frames per second for 1 filter iteration.

CUDA excels are performing high speed arithmetic operations, and whereas Lut, Merge and Transpose are memory bound and have trouble competing against 


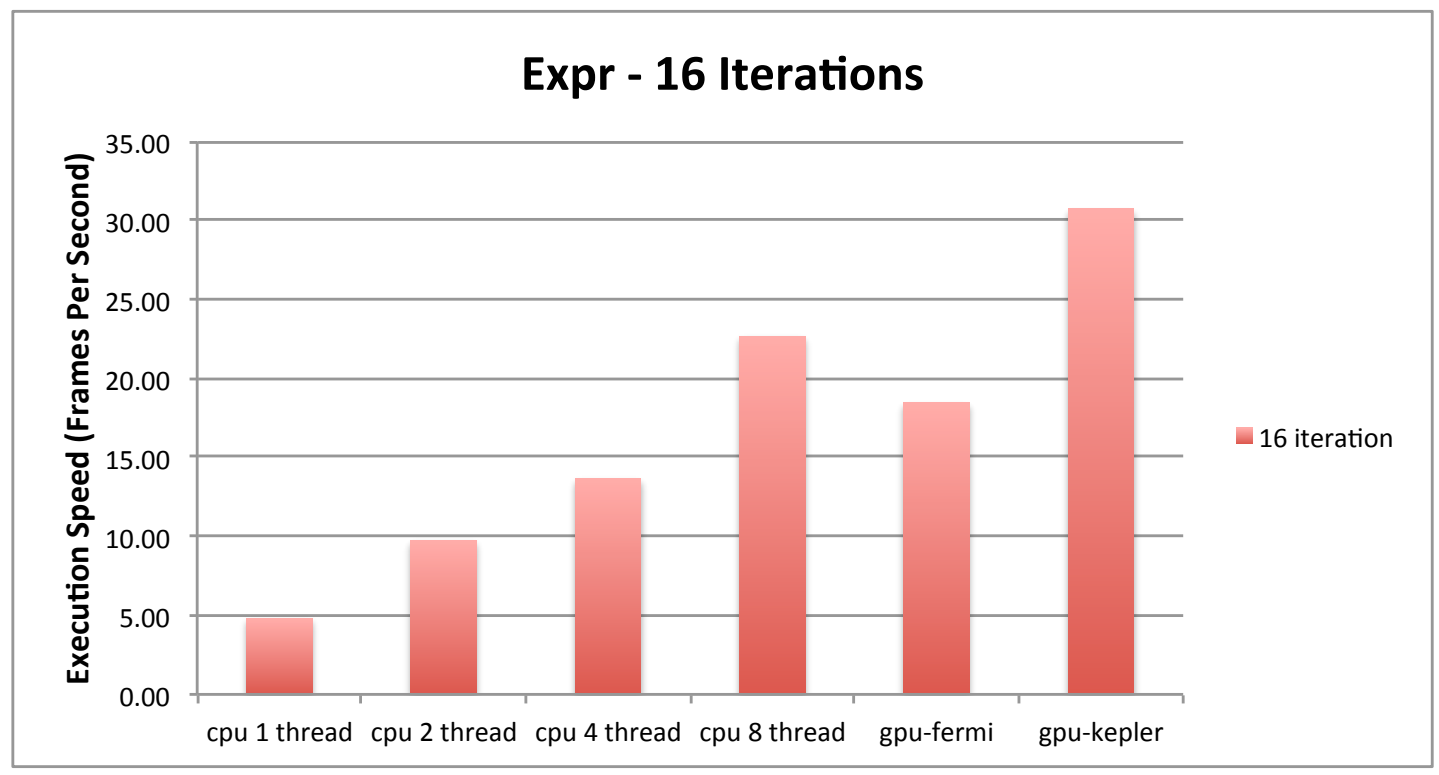

Figure 5.6: Expr execution speed in frames per second for 16 filter iteration.

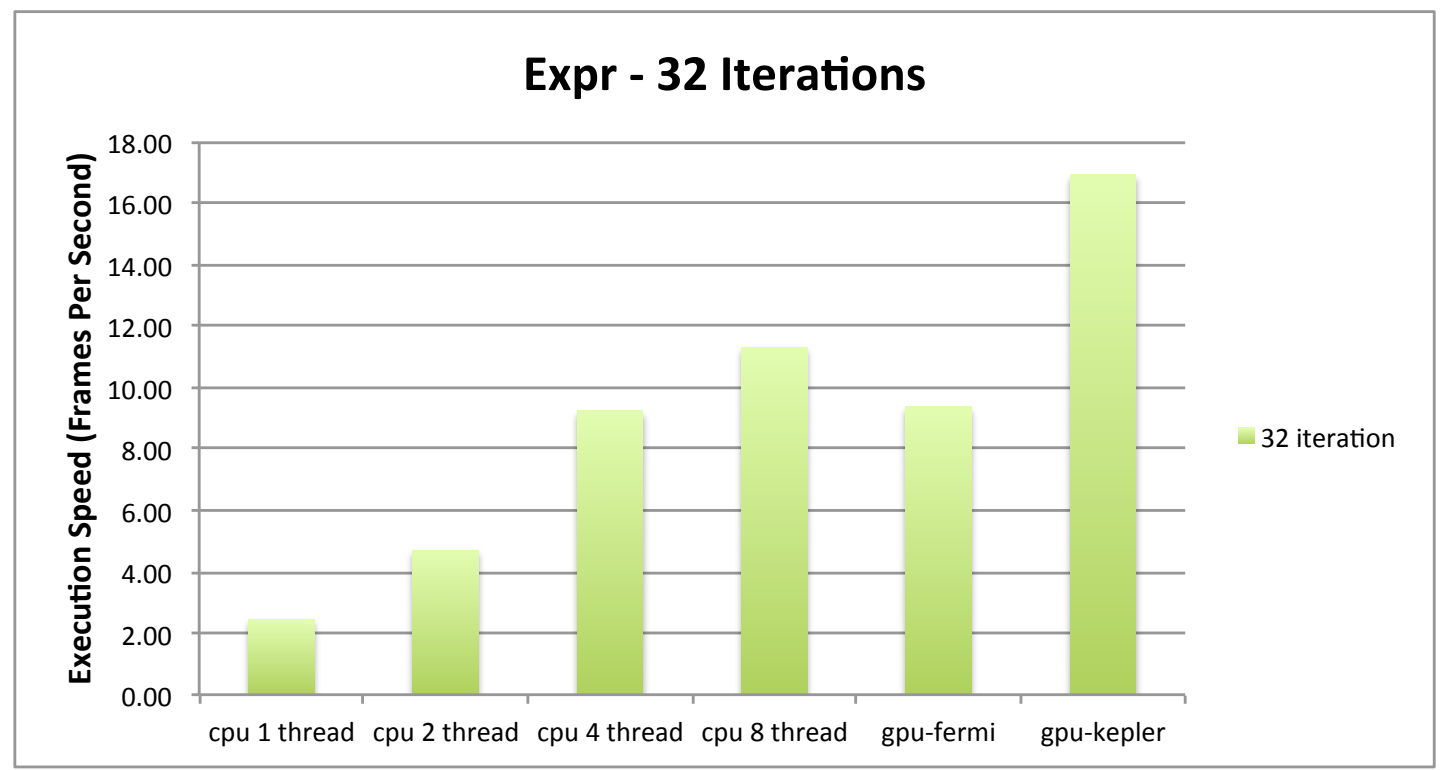

Figure 5.7: Expr execution speed in frames per second for 32 filter iteration.

the CPU, Expr excels over the CPU in all cases. This is further improved by enhancements to the Kepler architecture over the Fermi architecture for 16 and 
32 iteration runs.

As the next generation of CUDA, Kepler's raw compute performance significantly increased over the older Fermi architecture. With a new generation of the Streaming Machine and a much larger transistor count, Kepler offers the potential for a vast performance increase over all prior architectures. These additional Streaming Machines require a significant workload in order to produce measurable performance gains. Due to Expr's heavy emphasis on arithmetic computation it is a prime candidate for taking advantage of all of Kepler's new CUDA cores, much more so than the Lut, Merge, or Transpose filters.

For Expr, single iteration runs are almost too short to properly harness Kepler's added CUDA cores, but the 16 and 32 iteration runs offer enough workload to demonstrate a large performance boost over both the Fermi and CPU architectures. It would be very interesting to see performance numbers from NVIDIA's new GTX Titan or GTX 780 GPUs, both of which run off the latest GK110 Kepler chips. The only significant difference is the number of Streaming Machines available for CUDA computation, along with varying GDDR5 amounts and the use non-ECC enabled RAM.

\subsection{CUDA Stream Results}

The use of streams in a CUDA application allows for fully asynchronous communication between the host CPU and device GPU. Instead of kernel calls blocking the execution of a CPU thread, stream-enabled kernels return instantly and allow the CPU thread to continue with other work. CUDA streams are managed by dedicated hardware on devices with Compute Capability 2.0 and above. Kepler has a very special hardware management device referred to as Hyper-Q 


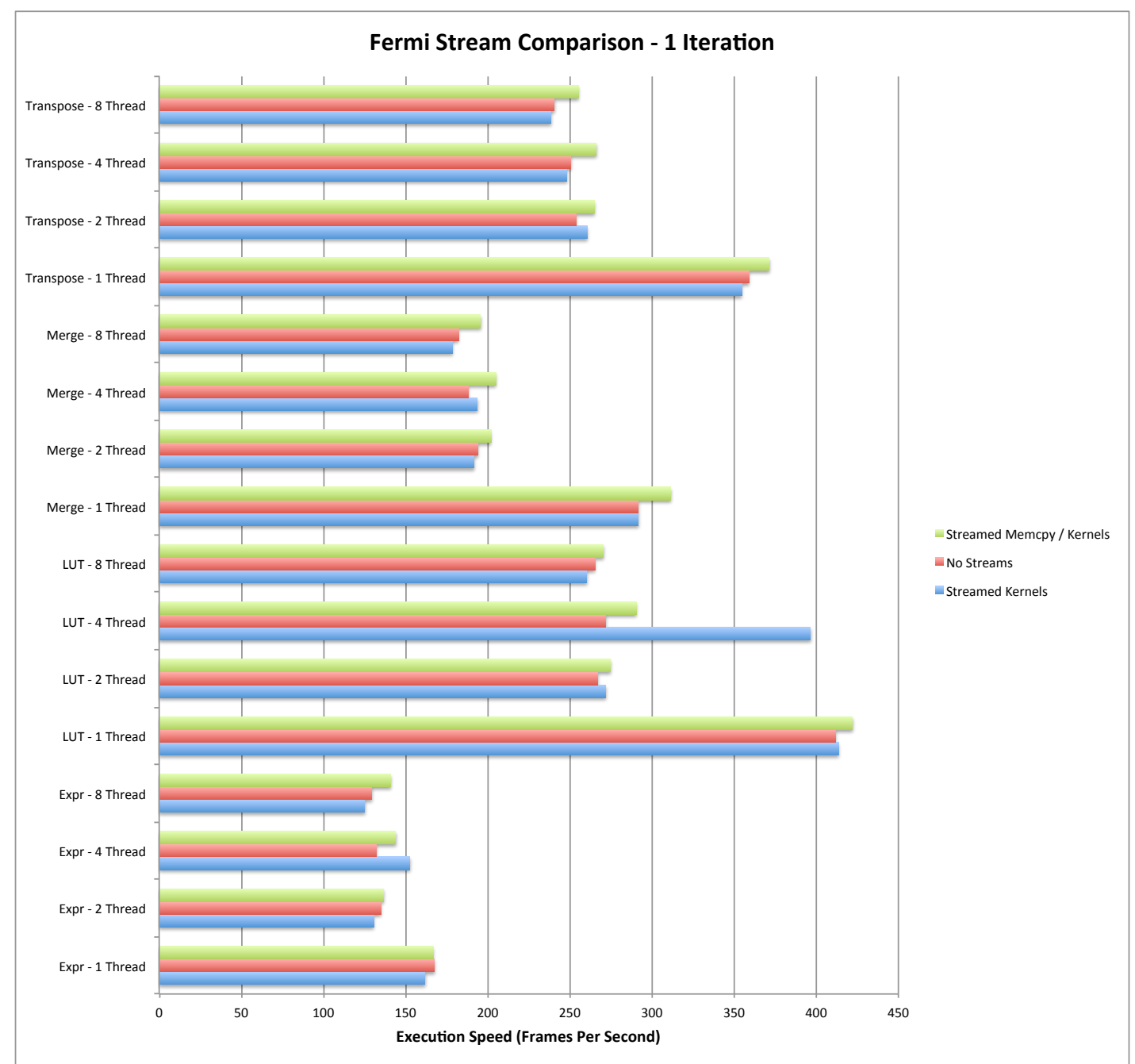

Figure 5.8: CUDA Streams results for one filter iteration on the Fermi architecture. Greater is better.

[19], which allows for each stream to be processed within its own hardware work queue, enabling complete computational independence between streams. Fermi offers only one hardware queue for all streams, which can introduce false serialization in a streamed application.

That being said, the current implementation of streams in Vapoursynth produces interesting results with a multithreaded CPU pipeline paired with a 16 (Fermi) or 32 (Kepler) streamed GPU pipeline. As stated in Chapter 4, all filter 
kernels are executed using CUDA streams, allowing their execution and memory copies to be completely asynchronous.

Figure 5.8 illustrates an important performance delta that streams offer during multithreaded CPU execution. More specifically, stream-enabled filter kernels demonstrate a distinct performance boost with four CPU threads when comparing against stream-disabled filter kernels. The Expr, Lut, and Merge kernels display the performance delta markedly so, with the Transose kernel showing little difference between streamed vs. non-streamed execution. Asynchronous memory transfers appear to make the most significant performance difference, with a fully asynchronous pipeline (memory copies and kernel executions) achieving the greatest performance. It is important to note that streams have the greatest effect on execution times when running with multiple CPU threads. The 2, 4, and 8 thread executions display a discernible gap between their non-streamed counterparts.

Its not quite clear why Expr and Lut display such a significant performance peak when executing with only streamed kernels and non-streamed memory copies while using multiple CPU threads. This is an area of interest for future research.

While the Fermi architecture shows some improvement through the use of streamed kernels and memory copies, Kepler's new HyperQ technology produces a significant performance improvement. Figure 5.9 presents the performance of non-streamed kernels, only streamed kernels, and streamed kernels with streamed memory copies. The latter approach (which was made possible by assigning streams on a per-plane basis and enabling fully asynchronous transfers) leads to a discernible boost in execution speed. 


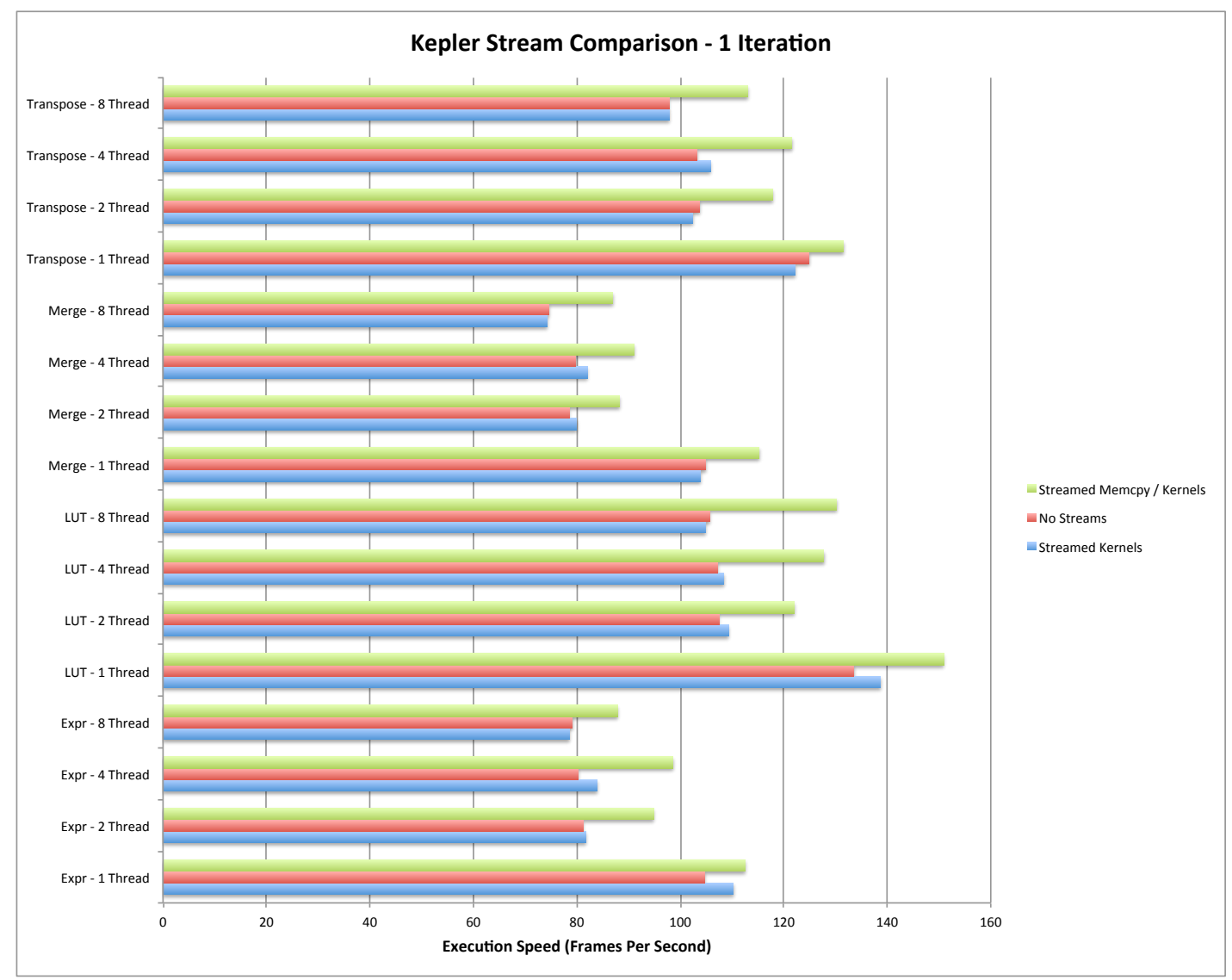

Figure 5.9: CUDA Stream results for one filter iteration on the Kepler architecture. Greater is better.

Similar to the Fermi execution, the greatest benefits of a fully streamed pipeline are seen when utilizing multiple CPU threads. This is likely due to the fact that CUDA allows for simultaneous memory copies, provided they operate in opposite directions. With a single CPU thread, bidirectional memory copies are impossible given that only one frame is pushed through the filter pipeline at a time. With multiple CPU threads, the potential increases significantly due to handling multiple frames simultaneously. These bidirectional, simultaneous memory copies are directly observable through the NVIDIA Visual Profiler, which reports a variety of important kernel execution metrics. 


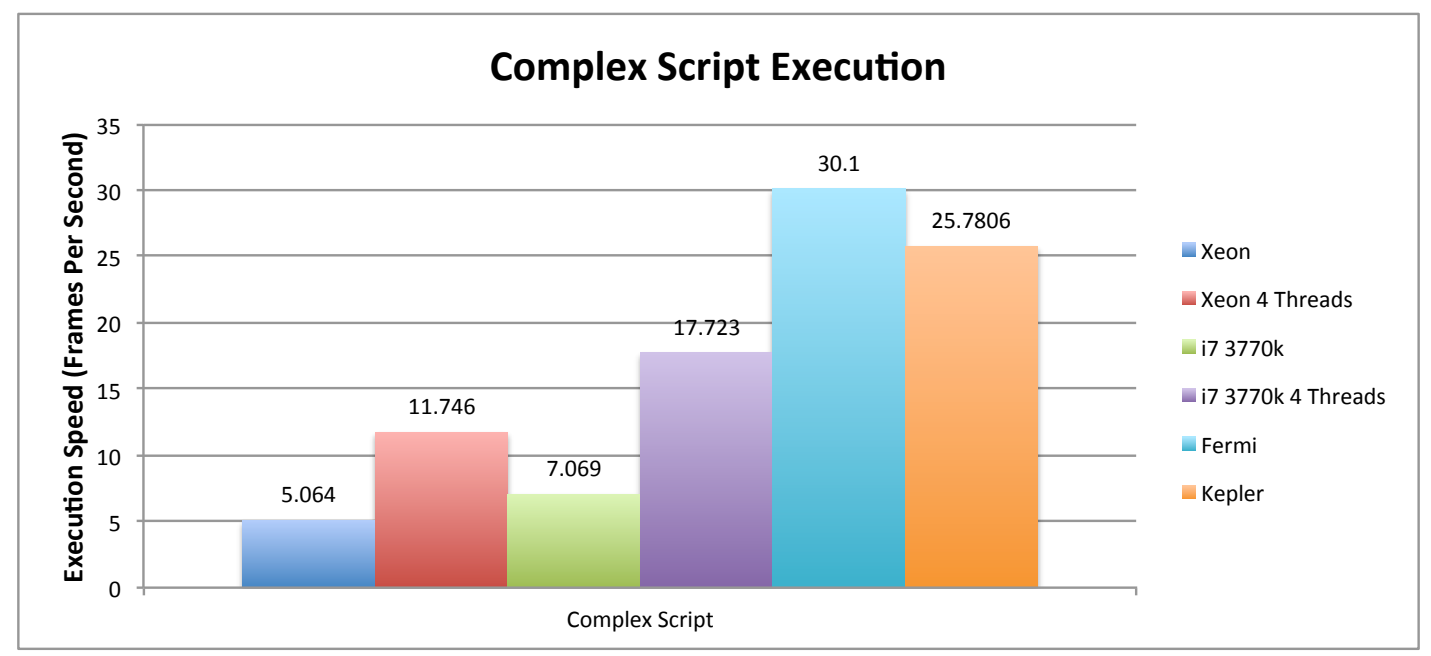

Figure 5.10: The results of a complex script run on an i7 Xeon E5-2650, i7 3770k, GTX 560 Ti, and Kepler K20Xm GPU.

\subsection{Complex Script Results}

In order to provide a more "real world" test for the extended CUDA pipeline, a script was devised that utilizes multiple GPU-capable filters. This script makes multiple calls to the Expr, Merge, and Transpose filters in order to instantiate a computationally complex filter chain. This mix of filters and additional complexity provides an excellent performance test for CPU and GPU filtering platforms.

The script was run on two different CPUs and two different GPUs. The Xeon processor is an i7 Xeon E5-2650 running at 2.00Ghz with a 2.8Ghz Turbo boost. The i7 $3770 \mathrm{k}$ is running at $3.5 \mathrm{Ghz}$ with a $3.9 \mathrm{Ghz}$ Turbo boost, and the Fermi and Kepler cards are detailed above. Figure 5.10 illustrates the execution speed of each run, with the CPUs utilizing one or four CPU threads. All GPU executions were run with one CPU thread. The script differs from previous performance tests by using a $4 \mathrm{k}$ resolution input clip, whereas earlier tests were limited to $2 \mathrm{k}$, or 1920 x 1080 pixels. This was done to demonstrate the performance improvement of a GPU pipeline as video resolutions continue to increase. 
The complex script is a very important performance metric, as it illustrates the raw power of a pure GPU filter pipeline through its ability to transfer data to the GPU and keep it there. None of the CPU runs are able to produce greater than 18 frames per second, which is below the standard 24 frames per second of realtime video. On the other hand, all GPU executions are able to filter frames at greater than realtime, with roughly 30 frames per second for Fermi and 25 frames per second for Kepler. In short, a GPU enabled pipeline is able to filter video at a framerate greater than realtime using only one CPU thread, while a CPU pipeline is hard pressed to meet 18 frames per second using four CPU threads, which is well below the realtime framerate threshold.

\subsection{Fermi and Kepler}

Some of the above results are surprising, given that Kepler is supposed to be an improved GPU architecture over the older Fermi. The decreased performance results recorded during testing may be the direct result of the Kepler K20Xm's use of ECC memory, while also being targeted at high precision scientific applications. The GTX 560 Ti Fermi card is targeted towards raw graphics performance and thus has shorter kernel startup times as well as no ECC memory. Future work is intended to test the GPU framework on graphics cards such as the GTX 780, which sports the newer GK110 Kepler architecture while still being targeted at high performance graphics processing instead of scientific computation. 


\section{Chapter 6}

\section{Conclusion}

CUDA enhanced video processing offers several benefits over traditional CPUbased video processing. In particular, video filters consisting of many arithmetic computations see a significant performance benefit when run on massively parallel, throughput-oriented GPUs.

However, several algorithm design characteristics must be observed in order to obtain optimal filter performance. More specifically, coalesced memory transactions are crucial for all filter implementations, especially for memory-bound filters requiring several memory reads for one memory write. In addition, the efficient use of constant or shared memory on the GPU can result in a significant performance speedup when compared to a global memory-only filter. This performance difference is best demonstrated through the Expr and Tranpose filters, which use the broadcast capabilities of constant memory and the extreme access speeds of shared memory, respectively. Additionally, a fully asynchronous execution pipeline is crucial for obtaining maximum performance while running in a multithreaded CPU environment. 
All in all, a completely GPU-enabled filtering pipeline is a viable design target for video processing frameworks like Vapoursynth, and can result in both saved CPU cycles and a tremendous performance improvement for computebound video filters. 


\section{Chapter 7}

\section{Future Work}

While the current implementation has proven to be a strong and stable platform for GPU video processing, several enhancements are left for future work.

\section{$7.1 \quad$ Multi-GPU Support}

The current implementation relies on multiple GPU streams for parallel kernel processing, but the possibility for a much greater speedup exists in systems with multiple CUDA-capable GPUs. By striping the workload across two or more GPUs, two frames can easily be processed in parallel, no matter how complicated the kernel. This contrasts with GPU streams, which must share resources between all streams running on the GPU and thus do not guarantee complete parallel processing. 


\subsection{Extended Filter Support}

After finishing the standard library of core filters, several other Vapoursynth plugins show strong potential for porting to the GPU. In particular, the Generic Filters [13] plugin, which implements a large number of mask-based operations, a la Masktools for Avisynth, should see a particularly large speedup when paired with an all GPU filtering chain. A number of its basic filters, including horizontal and vertical convolution, sobel edge filtering, and local average inflation/deflation, are already implemented in a basic form in NVIDIA's NPP library [17]. A proper evaluation of the NPP library against a custom implementation is definitely needed.

One caveat of using the NPP library is that it does not natively support video with a bit depth greater than 8 bits, which is a basic requirement for a capable plugin contribution to the Vapoursynth project.

\subsection{Expanded Bits Per Pixel support}

In the interest of time, all filters ported to CUDA so far have been limited to support only 8 bits per pixel. This is by far the most common video format, which means that these filters will work stably with almost all consumer video. However, in the interest of being feature complete, these filters need to support all standard bits per pixel video formats, including 9/10/16-bit and floating point. 


\subsection{Investigate CPU Threading problems}

Currently, when using more than one CPU thread for GPU filtering in Vapoursynth, performance takes a significant hit depending on the filter chain. Further research needs to be conducted into why this is and ways to improve performance in a multithreaded environment.

\subsection{Providing Support for Non-CUDA Devices}

The definitive problem with a CUDA-backed Vapoursynth project is that it is by definition limited to only CUDA-capable devices. At the time of this writing, this means that only NVIDIA sanctioned devices are able to run the kernels written in this project fork.

A direct port to OpenCL is a possible solution, but poses a few problems. Specifically, the complete rewrite and duplication of code just for extended device support.

Another option is offered through the GPU Ocelot [2] project, which provides the ability to perform a low-level PTX assembly conversion to support alternative devices such as ATI's GPUs or the recent Intel MIC (Knight's Corner) project. The benefit of using Ocelot is that there is no source code rewrite required, and it essentially allows a direct binary conversion. The downside is that Ocelot is still a very young project and can be difficult to setup and configure. 


\section{Bibliography}

[1] An example of color banding. https://commons.wikimedia.org/wiki/ File\%3AColour_banding_example01.png.

[2] Gpu ocelot: A modular dynamic compilation framework for heterogeneous systems. https://code.google.com/p/gpuocelot/.

[3] Msi gtx 560 ti hawk. http://www.techpowerup.com/gpudb/b936/ msi-gtx-560-ti-hawk.html.

[4] Luajit bindings for vapoursynth. https://github.com/tgoyne/luasynth, 2012.

[5] Apple. Video sample rate and bit depth. http://documentation.apple. com/en/finalcutpro/usermanual/index $\cdot \mathrm{html} \#$ chapter $=\mathrm{C} \% 26$ section= $11 \% 26$ tasks=true.

[6] I. Brabham. Avisynth version 2.6. http://avisynth.org/mediawiki/ Changelist_25-26.

[7] I. Corportation. Ia-32 intel architecture software developer's manual. Intel Corportation, 2001.

[8] Equays. Color formats for image and video processing. http://www. equasys.de/colorformat.html. 
[9] A. M. D. Inc. Amd extensions to the 3dnow! and mmx instruction set manual. Technical report, March 2000.

[10] F. Melbin. Vapoursynth video processing framework. http://www. vapoursynth.com/about/, 2012.

[11] F. Melbin. Expr - vapoursynth filter. http://vapoursynth.com/doc/ functions/expr.html, 2013.

[12] S. Moore. Using streaming simd extensions (sse2) to perform big multiplications. application note ap-941, intel corporation, 2000. version 2.0. Order, (248606-001).

[13] O. Motofumi. Vapoursynth generic filters. http://forum.doom9.org/ showthread. php?t=166842, 2013.

[14] Nand. Hi10p info / guide. http://haruhichan.com/wpblog/index.php/ 205/hi10p-info-guide.html, July 2011.

[15] NVIDIA. Nvidia cuda compute capabilities. http://docs.nvidia.com/ cuda/cuda-c-programming-guide/index.html\#compute-capabilities.

[16] NVIDIA. Nvidia kepler k20xm. http://www.techpowerup.com/gpudb/ 1884/tesla-k20xm.html.

[17] NVIDIA. Nvidia performance primitives. https://developer.nvidia. com/sites/default/files/akamai/cuda/files/CUDADownloads/NPP_ Library.pdf.

[18] NVIDIA. Nvidia cuda c programming guide version 5 . http:// docs.nvidia.com/cuda/cuda-c-programming-guide/index.html, October 2012 . 
[19] NVIDIA. Nvidia's next generation cuda compute architecture: Kepler gk110. http://www.nvidia.com/content/PDF/kepler/ NVIDIA-Kepler-GK110-Architecture-Whitepaper .pdf, 2012.

[20] NVIDIA. Tuning cuda applications for kepler. http://docs.nvidia.com/ cuda/kepler-tuning-guide/index.html, 2012.

[21] NVIDIA. Cuda: Coalesced access to global memory. http: //docs.nvidia.com/cuda/cuda-c-best-practices-guide/index. html\#coalesced-access-global-memory, 2013.

[22] NVIDIA. Parallel programming and computing plaform — nvidia cuda. http://www.nvidia.com/object/cuda_home_new.html, June 2013.

[23] B. Rudiak-Gould. Avisynth. http://avisynth.org/mediawiki/Main_ Page, 2003.

[24] SEt. Avisynth $2.6 \mathrm{mt}$. http://forum.doom9.org/showthread.php?t= 148782, 2013.

[25] B. Waggoner. Compression for great digital video: power tips, techniques, and common sense. Focal Press, 2002. 